\title{
Orientação Parental para Insônia Infantil em Irmãos Gêmeos: Um Relato de Caso
}

\author{
Renatha El Rafihi-Ferreira ${ }^{1}$ \\ ${ }^{1}$ Universidade de São Paulo, SP, Brasil.
}

\author{
Maria Laura Nogueira Pires ${ }^{2}$ \\ ${ }^{2}$ Universidade Estadual Paulista Júlio de Mesquita \\ Filho, SP, Brasil.
}

Edwiges Ferreira de Mattos Silvares ${ }^{1}$

${ }^{1}$ Universidade de São Paulo, SP, Brasil.

Resumo: A intervenção comportamental, por meio da orientação parental, vem alcançando resultados positivos no manejo da insônia em crianças. Contudo, questiona-se a efetividade de tais intervenções em irmãos gêmeos, pelo fato de os pais partilharem os cuidados de duas crianças da mesma idade e nível de desenvolvimento. O objetivo deste estudo é apresentar um relato de caso de intervenção comportamental para insônia infantil em irmãos gêmeos por meio de um programa dirigido aos pais e verificar o efeito da intervenção no sono e comportamentos diurnos das crianças. A mãe dos gêmeos foi orientada quanto as técnicas de extinção e reforço positivo no manejo do problema de sono infantil. Os resultados demonstraram que depois da intervenção houve melhora nos hábitos de sono, na qualidade do sono e nos comportamentos internalizantes e externalizantes das crianças. Os achados apresentados neste estudo mostraram os efeitos positivos que a intervenção baseada na aprendizagem operante teve sobre o sono e o comportamento infantil. Este aspecto ressalta a importância de uma inserção maior da questão do sono na Psicologia, a partir de práticas preventivas e interventivas.

Palavras-chave: Sono, Comportamento, Criança, Intervenção comportamental, Gêmeos.

\section{Parental Guidance for Childhood Insomnia in Twins: A Case Report}

\begin{abstract}
Behavioral intervention by means of parental guidance has been achieving positive results in the management of insomnia in children. However, the effectiveness of such interventions in twins is questioned because parents share the care of two children of the same age and level of development. This is a case report of behavioral intervention for childhood insomnia in twins through a program for parents. The objective of this study is to analyze the effect of such intervention on children's sleep and daytime behaviors. The mother of the twins was instructed on the techniques of extinction and positive reinforcement in the management of children's sleep problems. The results showed that after the intervention there was an improvement in the children's sleep habits, sleep quality and internalizing and externalizing behaviors. The findings of this case report show the positive effects that an intervention based on operant learning had on children's sleep and behavior. This highlights the importance of a greater inclusion of issues related to sleep in psychological studies based on preventive and intervention practices.
\end{abstract}

Keywords: Sleep, Behavior, Child, Behavioral intervention, Twins. 


\title{
Orientación Parental para El Insomnio Infantil en Hermanos Gemelos: Un Informe de Caso
}

\begin{abstract}
Resumen: La intervención comportamental, por medio de la orientación parental, viene alcanzando resultados positivos en el manejo del insomnio en niños. Sin embargo, se cuestiona la efectividad de tales intervenciones en hermanos gemelos, por el hecho de que los padres comparten los cuidados de dos niños de la misma edad y nivel de desarrollo. El objetivo de este estudio es presentar un relato de caso de intervención comportamental para insomnio infantil en hermanos gemelos por medio de un programa dirigido a los padres y verificar el efecto de la intervención en el sueño y comportamientos diurnos de los niños.. La madre de los gemelos fue orientada en cuanto a las técnicas de extinción y refuerzo positivo en el manejo del problema del sueño infantil. Los resultados demostraron que después de la intervención hubo mejoría en los hábitos de sueño, en la calidad del sueño y en los comportamientos internalizantes y externalizantes de los niños. Los hallazgos presentados en este estudio mostraron los efectos positivos que la intervención basada en el aprendizaje operante tuvo sobre el sueño y el comportamiento infantil. Este aspecto resalta la importancia de una inserción mayor de la cuestión del sueño en la psicología, a partir de prácticas preventivas e interventivas.
\end{abstract}

Palabras clave: Sueño, Comportamiento, Niño, Intervención comportamental, Gemelos.

A Insônia Comportamental Infantil pode ser definida como um problema de sono de ordem comportamental, caracterizado pela dificuldade para adormecer ou de permanecer dormindo ao longo da noite, despertando várias vezes, resistindo a voltar a dormir, especialmente sem a presença dos pais (Mindell, Kuhn, Lewin, Meltzer, \& Sadeh, 2006). Estudos de prevalência indicam que $30 \%$ da população infantil apresenta dificuldades com o sono, que podem ser decorrentes de associações inapropriadas com o processo de adormecer ou por dificuldades de imposição de limites por parte dos pais (Meltzer \& Mindell, 2014). A má qualidade do sono pode prejudicar a funcionalidade diurna e afetar aspectos comportamentais e emocionais da criança, além de estar associada ao estresse familiar (Tikotzky \& Sadeh, 2010). Vários estudos (Blunden \& Chervin, 2010; Byars, Yeomans-Maldonado \& Noll, 2011; Rafihi-Ferreira, Silvares, Pires, Assumpção \& Moura, 2016), nos quais a associação entre qualidade do sono e problemas comportamentais foi investigada, apontaram uma relação entre problemas externalizantes e internalizantes e problemas de sono em crianças. Devido aos prejuízos que os problemas de sono podem acarretar para o bem-estar da criança e família, justifica-se a necessidade de tratamento.

As abordagens de tratamento comportamental para crianças com dificuldade em iniciar e manter o sono são apoiadas pela literatura, devendo ser a primeira escolha terapêutica enfatizada pela Academia Americana de Sono (Thomas, Moore, \& Mindell, 2014). O tratamento para insônia infantil baseia-se na premissa de que as dificuldades para adormecer e os frequentes despertares noturnos estão relacionados com o envolvimento parental no momento do início do sono. Se os cuidadores auxiliam seus filhos a adormecer no momento de dormir, a criança pode não ter a oportunidade de desenvolver as habilidades de auto regulação necessárias para acalmar-se independentemente. Por tais motivos, a intervenção tem como foco a mudança de comportamento dos pais (Thomas et al., 2014). Esta, consiste em uma capacitação para pais em estratégias comportamentais (modelagem, reforço, extinção) baseadas nos princípios de aprendizagem operante. A capacitação envolve um treino terapêutico para os pais se tornarem agentes ativos na mudança dos comportamentos problemáticos relacionados ao sono de suas crianças (Mindell et al., 2006). As intervenções comportamentais apoiadas empiricamente no contexto dos problemas de sono infantil incluem educação parental sobre o sono da criança, com informações sobre estabelecimento de rotinas pré-sono, reforço positivo e extinção. Entre os tratamentos comportamentais para crianças pequenas e pré-escolares, nenhum outro tratamento 
foi mais investigado ou amplamente aplicado como a orientação parental. Por esta razão a revisão (Mindell et al., 2006) realizada por um grupo apoiado pela Academia Americana de Medicina do Sono indica as estratégias de extinção e estabelecimento de rotinas que ocorrem por meio de educação parental, no tratamento dos problemas de sono em crianças pequenas e em idade pré-escolar. Os procedimentos envolvidos na orientação parental estão descritos a seguir:

Rotinas pré-sono envolvem um conjunto de atividades tranquilas que antecedem o momento de dormir. Os pais são orientados a estabelecerem atividades relaxantes que devem ocorrer todas as noites em uma mesma ordem, em um período de 30 a 40 minutos. Essas atividades podem incluir, por exemplo, banho, livro de história, oração e cama. A escolha das atividades também deve respeitar a cultura familiar em que a criança está inserida (Meltzer \& Mindell, 2011).

Para o estabelecimento de rotina, os pais são orientados quanto à utilização da técnica do reforço positivo para ensinar a criança comportamentos apropriados em relação ao sono. Os reforçadores devem ser contingentes aos comportamentos apropriados durante a noite. A rotina pré sono deve ser programada por meio de comportamentos que indicam o momento de dormir, como escovar os dentes, vestir o pijama, ir para o quarto, se deitar, escutar uma história e relaxar. Desta forma, as atividades devem finalizar no ambiente em que a criança dorme. Na execução da técnica, os pais são orientados a reforçar (por meio de atenção, carinho, elogios, brinquedo) o comportamento adequado da criança (ficar quieto, não chorar, permanecer na cama) na rotina pré-sono e momentos antes de dormir (Didden, Sigafoos, \& Lancioni, 2011; Kuhn, 2011). Neste sentido, os pais são orientados a não reforçarem os comportamentos inadequados (chorar, protestar) da criança (Didden et al., 2011; Kuhn, 2011).

A técnica de extinção pode ocorrer de forma sistemática, gradual e na presença dos pais e sua execução somente acontece na hora de dormir. A extinção sistemática consiste em colocar a criança na cama e ignorar seus protestos (choros, solicitações e birras) até o horário designado para a criança acordar. Exceções para não ignorar o comportamento inadequado da criança incluem situações em que a criança pode se machucar ou quando a criança está doente. O maior obstáculo na execução da técnica é a inconsis- tência parental. Se os pais fornecerem atenção para a criança depois de determinado tempo ou de vez em quando, reforçarão intermitentemente o comportamento inadequado da criança, de forma que ela aprenderá a chorar mais nas próximas ocasiões. Esse procedimento configura-se como uma técnica muito estressante para os pais. Muitos não são capazes de ignorar os protestos por tempo suficiente para que a intervenção seja eficaz. Por essa razão, alguns estudos passaram a utilizar variações da técnica de extinção, ou seja, a extinção na presença dos pais ou a extinção gradual.

A extinção na presença dos pais tem a mesma metodologia da extinção sistemática, tendo como diferencial a presença dos pais. Nessa variação, os pais permanecem no quarto ou próximos à criança, contudo ignoram o comportamento inadequado do(a) filho(a). Nesta modalidade de extinção, os pais são instruídos a não interagirem com a criança. Muitos pais optam por esta técnica por apresentarem dificuldades em ficar longe da criança ou ficam excessivamente preocupados com o que possa ocorrer em sua ausência (Mindell et al., 2006).

Outra variação do procedimento de extinção é denominada extinção gradual. Nesta variação, os pais são instruídos a ignorar os protestos da criança, isto é saírem do quarto do(a) filho(a), podendo retornar para verificar a criança em períodos específicos (intervalo fixo - a cada 5 minutos ou horários progressivos, aumentando gradualmente o intervalo de tempo entre uma verificação e outra). Esta técnica permite que a criança se acostume com a ausência dos pais de maneira gradual e se autoacomode na ausência parental. A duração de tempo durante o qual os protestos são ignorados e o intervalo entre as verificações são adaptados de acordo com a idade e temperamento da criança, e também com a capacidade de tolerância dos pais frente aos protestos da criança. Os pais são orientados a minimizar as interações com a criança durante as verificações, pois a atenção pode reforçar o comportamento inadequado da criança. Esse procedimento tem como vantagem a verificação da criança, o que muitas vezes serve de conforto e segurança para os pais (Meltzer \& Mindell, 2011). As diferentes técnicas de extinção são igualmente eficazes, contudo, comparadas a extinção sistemática, na extinção gradual e na extinção na presença dos pais há uma demora maior para os resultados surgirem (Mindell et al., 2006). 
A eficácia da terapia comportamental para insônia em crianças pré-escolares já foi reportada em vários estudos (Meltzer \& Mindell, 2014; Mindell et al., 2006). Meltzer e Mindell (2014) conduziram uma revisão sistemática com o objetivo de avaliar e quantificar a evidência das intervenções comportamentais para insônia pediátrica, a partir da meta-análise de 16 estudos controlados, sendo 12 estudos com 1.874 crianças típicas na faixa etária entre zero e cinco anos. As análises consideraram as variáveis "latência para início do sono", "despertares noturnos" e "eficiência do sono". Os resultados desta revisão demonstraram moderado nível de evidência para o tratamento comportamental para insônia em crianças pequenas e em idade pré-escolar.

Um estudo recente realizado no Brasil por Rafihi-Ferreira, Pires e Silvares (2019) avaliou a eficácia da intervenção comportamental para problemas de sono em 62 crianças com idade pré-escolar e investigou os efeitos sobre o comportamento diurno. O programa de intervenção foi composto por cinco sessões nas quais os pais receberam educação sobre o sono da criança, orientações sobre o estabelecimento de horários e rotina para dormir e quanto ao uso de técnicas de extinção e reforço positivo para a melhoria do momento de dormir e redução de despertares noturnos. Os resultados mostraram que além da melhora no sono da criança, após a intervenção houve redução dos escores dos problemas de comportamento internalizante e externalizante.

Apesar de a literatura ressaltar a eficácia da intervenção comportamental, não se sabe se esses resultados seriam igualmente alcançados quando se trata de mais de uma criança com problema de sono. Desta maneira, a generalização dos resultados de pesquisas para crianças gêmeas pode sofrer limitações devido às diferenças entre amostras de gêmeos e não gêmeos. Uma das diferenças entre as amostras, é que crianças gêmeas apresentam algumas experiências de vida particulares. A relação parental entre as crianças e os cuidadores podem ser diferentes entre crianças gêmeas e não gêmeas, pois os pais, no primeiro caso têm que partilhar seus cuidados e atenção entre duas crianças da mesma idade e nível de desenvolvimento, o que não acontece no segundo (Oord, Koot, Boomsma, Verhulst, \& Orlebeke, 1995). Para Lytton, Singh e Gallagher (1995) a educação de filhos gêmeos submete os pais a um estresse especial que testam os limites das habilidades de educar filhos.
A rotina com gêmeos é mais estressante, devido aos cuidados intensos que ter duas crianças requer. Além disso é frequente cuidadores de gêmeos cuidarem dos dois filhos do mesmo modo, com horários semelhantes para banho, alimentação etc., o que implica divisão de cuidados. Uma revisão sistemática realizada por Andrade, Martins, Angelo e Martinho (2014) com objetivo de explorar a experiência parental de famílias com gêmeos encontrou que as mães de gêmeos apresentaram maiores níveis de estresse, exaustão e depressão, caracterizando uma experiência mais exigente e complexa comparada a experiência de mães que não tiveram filhos gêmeos. Os resultados desta revisão apontaram que entre os estressores presentes em famílias com gêmeos encontra-se a sobrecarga de atividades na realização de rotinas familiares e consequente privação de sono. A rotina diária demanda maior atenção e cuidado para os cuidadores de filhos gêmeos. Além disso, mães de gêmeos demoram um tempo maior para retornar as suas atividades independentes, como por exemplo o retorno ao trabalho, do que mães de crianças singulares, o que acaba interferindo em questões de independência e identidade. Todos esses aspectos podem contribuir no contexto social e psicológico dessas cuidadoras influindo de forma negativa em seu bem-estar.

Ao considerar que o vínculo de apego entre mãe e filho depende da qualidade dos cuidados parentais, ter gêmeos significa dividir esta atenção e dedicação. Segundo David, Azevedo, Russi, Bethoud e Oliveira (2000) o vínculo entre filhos gêmeos, se estabelece entre a tríade, isto é, com o cuidador e com cada um dos gêmeos, o que significa que o cuidador se vincula a ambas as crianças e que estas por sua vez se vinculam ao cuidador e ao irmão. Lytton et al. (1995) apontam que, ao comparar gêmeos e não gêmeos, as crianças gêmeas mostram maior apego inseguro, que pode ser explicado devido a excessiva demanda dos filhos quanto aos cuidados maternos.

Em relação a estudos sobre o sono infantil, há poucas investigações incluindo irmãos gêmeos. Um dos poucos estudos dessa natureza é o desenvolvido por Brescianini et al. (2011) com 314 pares de gêmeos, sendo 127 pares monozigóticos e 187 pares dizigóticos, com 18 meses de idade. O estudo demonstrou que a variação nos padrões de sono era predominantemente devido a fatores ambientais compartilhados. Na mesma direção, Fisher, Jaarsveld, Llewellyn e Wardle (2012) examinaram influên- 
cias genéticas e ambientais nas dificuldades com o sono em 3.730 crianças gêmeas, sendo 1.190 monozigóticos e 2.540 dizigóticos com idade média de 15 meses e os resultados apontaram que tanto influências genéticas como ambientais interferem no sono infantil, embora sobre todos os parâmetros do sono avaliados, a influência do ambiente compartilhado foi mais forte. Esses resultados chamam a atenção para a importância do ambiente familiar partilhado no desenvolvimento de intervenções.

Ao considerar que ambiente, rotina e relação parental desempenham papel fundamental para melhora do sono (Gregory, Eley, O'Connor, Rijsdijk, \& Plomin, 2005), é possível supor que se tratando de duas crianças, ao mesmo tempo, as dificuldades que as mães enfrentariam seriam maiores, o que poderia inviabilizar que o programa fosse conduzido com êxito. Desta forma, questiona-se se a orientação parental para o manejo da insônia infantil também seria efetiva quando se trata de insônia comportamental em gêmeos.

O presente estudo tem o objetivo de apresentar um relato de caso de intervenção comportamental para insônia infantil em irmãos gêmeos por meio de um programa dirigido aos pais e verificar o efeito da intervenção no sono e comportamentos diurnos das crianças.

\section{Método}

\section{Caracterização clínica do caso}

A intervenção foi realizada com um par de gêmeos monozigóticos, nascidos com 37 semanas, do sexo masculino com dois anos de idade e sua mãe. Os pais dos gêmeos eram casados, ambos tinham escolaridade superior completa e eram do estrato social B2 (Critério Brasil). A mãe das crianças tinha 26 anos e era dona de casa e o pai tinha 34 anos e trabalhava na área da Saúde.

De acordo com o Inventário de Autoavaliação para Adultos de 18 a 59 anos (ASR) a mãe não apresentava escores clínicos para problemas de comportamento internalizante, externalizante e para total de problemas de comportamento.

Os gêmeos residiam com seus pais na mesma casa que tinha dois quartos, um banheiro, sala e cozinha. No momento do estudo e de acordo com o relato materno, os irmãos não frequentavam a pré-escola e não apresentavam outros problemas de saúde. O relato da mãe sobre os comportamentos dos gêmeos e as respostas aos questionários e diário de sono indicavam a presença de problemas de ordem comportamental relacionados ao sono, descritos como: protesto e resistência em ir para cama, demorar mais de 30 minutos para adormecer, despertar durante a noite e dormir apenas na presença dos pais. Para ilustrar o caso, as crianças serão chamadas de J.B. e E.

Local da intervenção: Clínica-escola de uma universidade pública.

\section{Procedimentos de avaliação}

A intervenção foi conduzida pela primeira autora que é psicóloga e mestre em Análise do Comportamento e na ocasião era doutoranda em Psicologia Clínica, na Universidade de São Paulo. As sessões de avaliação e intervenção foram realizadas individualmente com a primeira autora e a mãe das crianças. Os participantes foram avaliados em quatro momentos específicos, no período pré-tratamento, uma semana antes da intervenção, no período pós-tratamento, uma semana após a intervenção e no período de seguimento de um e seis meses após a intervenção.

\section{Instrumentos de avaliação}

Diário de Sono e de Comportamentos: Trata-se do registro de características e padrões de sono das crianças, dos comportamentos das crianças ao se deitarem e ao despertarem durante a noite e dos comportamentos dos cuidadores ao lidarem com a situação. Para acessar essas informações, a mãe foi orientada a registrar as ações das crianças ao se deitar e ao despertar durante a noite, bem como as suas próprias respostas frente à situação. Tais registros auxiliam a determinar a extensão e a natureza dos problemas de comportamentos associado ao sono, como também os comportamentos relacionados à relação pais e filhos.

Escala UNESP de Hábitos e Higiene do Sono-Versão Crianças: Elaborada por Pires, Vilela, e Camara (2012), esta escala é composta de questões sobre os horários, rotina do sono, alerta fisiológico, aspecto cognitivo/emocional, conforto do ambiente de dormir, presença de dificuldades para dormir e sonolência diurna.

Índice Composto de Distúrbios de Sono (ICDS): Adaptado do estudo de Richman e Graham (1971), de 
forma a contemplar as principais variáveis de sono infantil e obter uma pontuação total. O índice compreende as variáveis "resistir a ir para a cama", "tempo para adormecer", "despertares noturnos" e "dormir com os pais", fornecendo uma pontuação que varia de 0 a 12, de maneira que, quanto maior a pontuação, maior o problema de sono.

Inventário de Comportamentos para Crianças entre $1^{1 / 2}$ a 5 anos (CBCL) (Achenbach \& Rescorla, 2000): Obtém taxas padronizadas de problemas comportamentais de crianças, caracterizando-as quanto a seu Funcionamento Global (clínico e não clínico) e nos perfis Internalizante e Externalizante. Os valores para análise dos escores T em total de Problemas de Comportamento, Perfil Internalizante e Perfil Externalizante classifica como clínico os escores iguais ou superiores a 60. Para a escala Problemas de Sono, os escores clínico são iguais ou superiores a 65.

\section{Procedimento de intervenção}

O programa de intervenção foi conduzido apenas com a mãe, sem a presença das crianças e foi composto por cinco sessões com duração de 60 minutos, sendo que as três primeiras realizadas semanalmente e as duas finais quinzenalmente. Durante as sessões, a mãe recebeu orientações sobre o estabelecimento de rotina para dormir e reforço positivo e quanto ao uso da técnica de extinção para a melhoria do momento de dormir e redução de despertares noturno da criança. Tais orientações estão descritas, a seguir:

Estabelecimento de rotina para dormir e reforço positivo: A mãe foi orientada quanto à utilização da técnica do reforço positivo para ensinar as crianças comportamentos apropriados em relação ao sono. $\mathrm{O}$ estabelecimento de rotinas antes de dormir deve ocorrer por meio de comportamentos que indicam o momento de dormir, como escovar os dentes, vestir o pijama, ir para o quarto, deitar-se, escutar uma história e relaxar. Na execução da técnica, a mãe foi orientada a sempre reforçar - por meio de atenção, carinho, elogios - os comportamentos adequados das crianças, isto é, aqueles comportamentos que favorecem o processo de adormecer, como ficar calmo, não chorar, permanecer na cama, no momento da rotina pré-sono e um pouco antes de dormir. Do mesmo modo, a cuidadora foi orientada a ter o cuidado de não reforçar os comportamentos inadequados (chorar, protestar) das crianças.
Extinção: A variação da técnica de extinção utilizada no presente estudo foi a extinção gradual. A mãe foi orientada, após concluir a rotina pré-sono junto as crianças, a ignorar os comportamentos inadequados dos filhos, ou seja, os comportamentos que concorrem com a acomodação necessária para o relaxamento. Nesta variação da técnica, os protestos devem ser ignorados por períodos específicos, isto é, nas primeiras noites durante cinco minutos, depois de algumas noites durante 10 minutos, aumentando gradativamente o intervalo de verificação no decorrer dos dias. Durante as verificações a orientação foi reduzir as interações com as crianças, a fim de não reforçar o comportamento inadequado. Neste momento, a cuidadora foi instruída a fornecer, se necessário, breves respostas verbais ("Estou aqui, vão dormir”), de modo que as verificações durassem em média de 15 a 60 segundos.

\section{Aspectos éticos}

Este estudo atendeu os procedimentos éticos adotados em pesquisas que envolvem seres humanos e foi aprovado pelo Comitê de Ética em Pesquisa (número CAAE: 05160812.2.0000.5561) onde foi realizada a intervenção.

\section{Resultados}

Os resultados foram divididos em: Comportamentos maternos e infantil no momento de dormir; Hábitos e rotinas das crianças antes de dormir; Variáveis do sono infantil; e Comportamento diurno infantil.

\section{Comportamentos maternos e infantil no momento de dormir}

Os comportamentos das crianças e da mãe no momento de dormir, coletados por meio do Diário de Sono e de Comportamentos, foram organizados, categorizados e distribuídos em frequências. Para melhor especificar os comportamentos das crianças que podem interferir no sono, os comportamentos foram categorizados em grupos de ações. Tais comportamentos referem-se a qualquer ação da criança que ocorreu após a rotina pré-sono e ao momento estabelecido para dormir. Os comportamentos foram divididos nas seguintes categorias: vocalizar (qualquer vocalização audível vinda da criança, como cantar, rir, chorar, falar, gritar, fazer pedidos, com a exclusão de 
espirros, tosse ou bocejos); sair da cama; permanecer deitada. Para fins de ilustração, apenas o comportamento de sair da cama será apresentado. Os comportamentos maternos referem-se às respostas que a mãe apresentou perante o comportamento das crianças no momento de dormir e no despertar noturno. Tais comportamentos foram classificados como contato físico, vocalização (som audível do cuidador direcionado diretamente à criança), comportamento de ignorar e comportamento de elogiar.

A Figura 1 demonstra a frequência dos comportamentos da mãe de vocalizar, de ter contato físico, de ignorar os comportamentos inadequados e de elogiar os comportamentos adequados das crianças no momento de dormir, bem como o comportamento das crianças de sair da cama, apresentado em porcentagem de noites em cada fase em que ocorreu o comportamento, considerando os períodos pré-tratamento, as cinco sessões, o período pós-tratamento e de follow-up de um e seis meses.

Houve mudanças substanciais na frequência dos comportamentos das crianças e da mãe. Com as orientações, a mãe reduziu a frequência de contato físico e vocalização com as crianças no momento de dormir, que era bem frequente no período pré-tratamento; passou a ignorar os comportamentos inadequados e a elogiar os comportamentos adequados para o sono. Paralelamente, os comportamentos de vocalização e sair da cama reduziram e as crianças passaram a permanecer deitadas na cama mais fre-
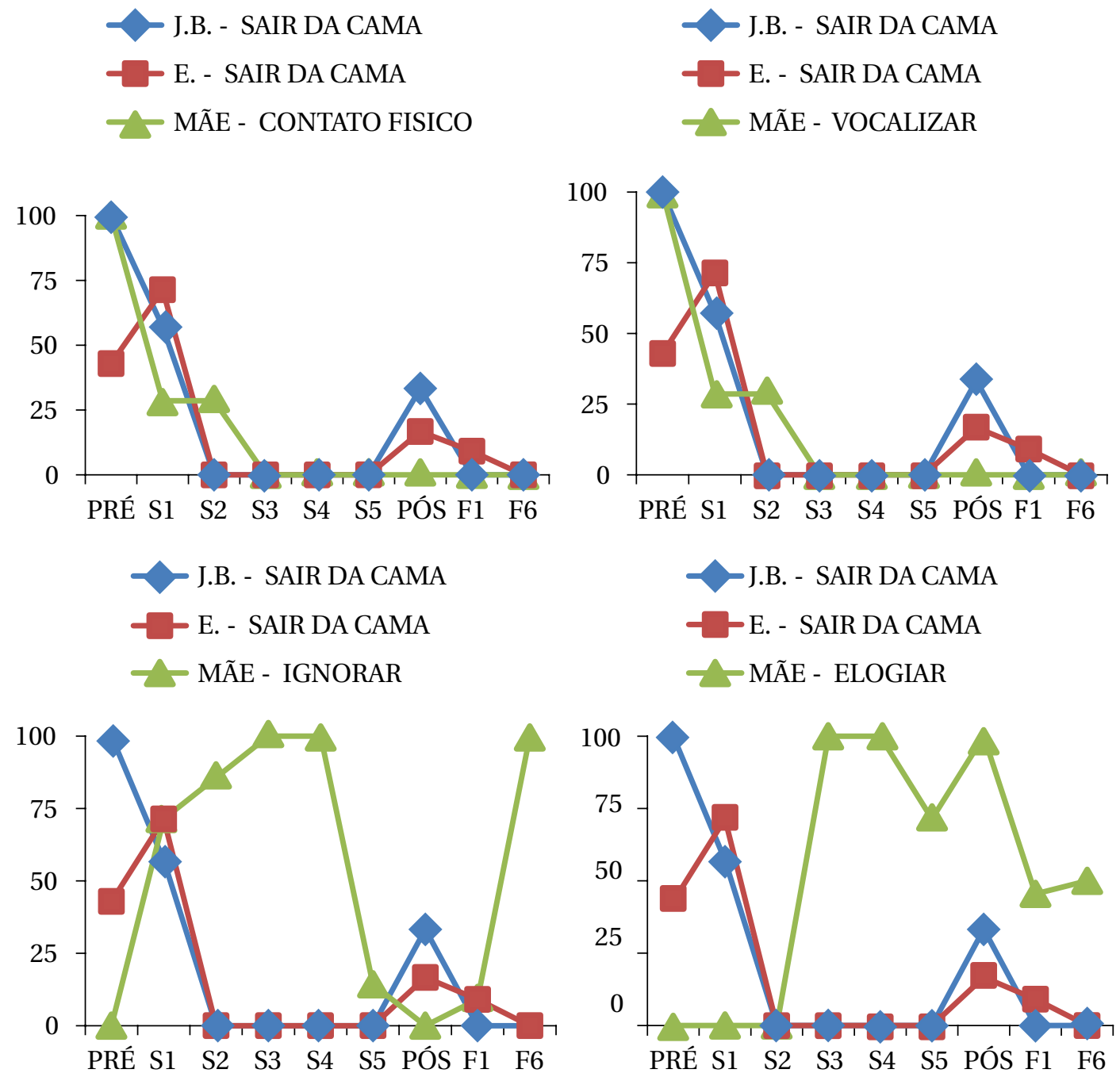

Figura 1

Comportamentos maternos (contato físico, vocalizar, ignorar, elogiar) frente ao comportamento de sair da cama das crianças, antes (Pré), durante (S1-S5) e após a intervenção (Pós, F1, F6). 
quentemente no momento de dormir. Como pode ser observado na Figura 1, a redução do comportamento de sair da cama, em ambos irmãos, manteve-se no período pós-tratamento (Pós) e nos períodos de seguimento (F1 e F6). Nota-se que o comportamento de E. de sair da cama aumentou em frequência, de $42 \%$ da primeira etapa (Pré) para 71\% na primeira sessão (S1), antes de reduzir nas subsequentes. Este fato deve-se a "explosão" de repostas que ocorrem no início do procedimento de extinção. Quando um comportamento inadequado é ignorado, a tendência é um aumento acentuado na frequência deste comportamento, antes da redução.

\section{Hábitos e rotina das crianças antes de dormir}

Os hábitos e rotina dos gêmeos foram avaliados pela Escala UNESP de Hábitos e Higiene do Sono-Versão Crianças. Antes da intervenção, os gêmeos demoravam mais de 30 minutos para adormecer e não tinham uma rotina pré-sono com atividades calmas. Todas as noites, antes de dormir eles assistiam TV e brincavam com atividades vigorosas, tais como correr atrás do outro, se esconder, pular etc. Quando chegava o momento de dormir, frequentemente (três ou mais vezes na semana) eles resistiam ir para a cama e apresentavam emoções fortes, como a raiva, antes de ir se deitar. Além disso, só adormeciam junto aos pais. O horário de ir para cama variava entre $22 \mathrm{~h} 50$ e $23 \mathrm{~h} 00$ e de acordar pela manhã entre $9 \mathrm{~h} 00$ e $9 \mathrm{~h} 30$, com duração do sono de aproximadamente 10 horas e meia.

Com a intervenção, a mãe dos gêmeos passou a estabelecer uma rotina pré-sono com atividades calmas como janta, banho e leitura antes de dormir. As crianças deixaram de resistir ir para cama, praticar atividades vigorosas, assistir TV e sentir emoções fortes, como raiva, antes de ir dormir. Além disso, os gêmeos passaram a adormecer mais rapidamente, isto é, em menos de 20 minutos, e dormir lado a lado em cama própria, não mais dividindo o espaço com os pais. $\mathrm{O}$ horário de ir para a cama passou a ser entre $22 \mathrm{~h} 00 \mathrm{e}$ 22h30, de acordar pela manhã permaneceu o mesmo horário, com duração total de sono de 11 horas. A mudança de horários, hábitos, rotinas e duração do sono foram mantidas até o período de seguimento de um mês. Contudo, na reavaliação do follow-up de seis meses, a mãe relatou que em algumas noites ela deixava de acompanha-los na rotina pré-sono com atividades calmas e começou a permitir que as crianças assistissem TV. Ademais, as crianças voltaram a resistir em ir para a cama, adormecer entre $22 \mathrm{~h} 40$ e $23 \mathrm{~h} 00$ e consequentemente tiveram uma redução no tempo total de sono, com duração de 10 horas e 15 minutos. Apesar de as crianças, no período de seguimento de seis meses, voltarem a dormir tarde e a resistir a ir para a cama, elas não mais dormiam com os pais, mantendo no período de seguimento, o hábito de dormir de forma independente.

\section{Variáveis do Sono Infantil}

As variáveis do sono dos gêmeos, foram avaliadas pelo ICDS, que apontou que antes da intervenção as crianças pontuaram escore máximo (12) no instrumento, indicando que resistiam frequentemente ir para a cama, demoravam mais de 30 minutos para adormecer, despertavam frequentemente a noite e adormeciam na companhia dos pais. Após a intervenção e no período de seguimento de um mês, os escores para o ICDS dos gêmeos, reduziram substancialmente, (2 para J.B. e 1 para E.) apontando melhora em todas as variáveis do instrumento (resistir ir para a cama, tempo para adormecer, despertares noturnos e dormir com os pais). No seguimento de seis meses, o escore do ICDS continuou baixo (3) para ambas as crianças, embora o escore do ICDS tenham apresentado um pequeno aumento, identificado na variável de resistência em ir para a cama. Desta forma, os gêmeos mantiveram a melhora em todas as variáveis do sono até o período de seis meses, com exceção da resistência em ir para a cama, que retornou no último período de seguimento. A evolução dos escores do ICDS no decorrer do estudo pode ser observada na Figura 2.

\section{Comportamento diurno infantil}

Os comportamentos diurnos avaliados pelo CBCL, no período pré-tratamento, indicaram escores clínicos, em problemas de sono $(100,94)$, comportamentos internalizantes $(62,67)$, comportamentos externalizantes $(65,76)$ e total de problemas de comportamento $(70,74)$, para J.B. e E. respectivamente. $\mathrm{Na}$ reavaliação pós-tratamento e de seguimento de um e seis meses, as crianças não mais pontuaram escores clínicos em nenhuma subescala do CBCL, apresentando escores abaixo de $60 \mathrm{em}$ todas as subescalas. Os escores do CBCL no decorrer da intervenção estão demonstrados na Figura 3. 


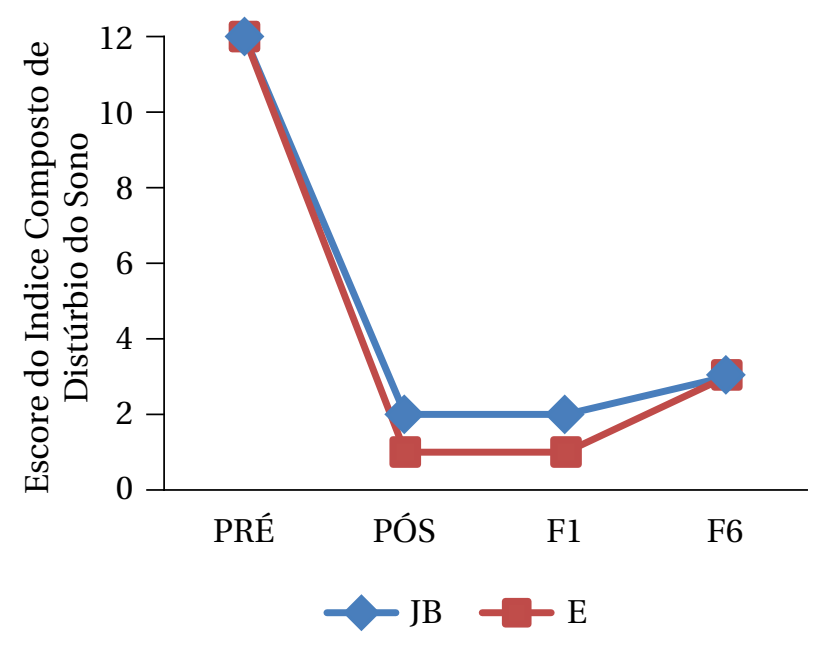

Figura 2

Escore do Índice Composto de Distúrbio do Sono dos gêmeos nos períodos pré, pós-intervenção e follow-up de um e seis meses.
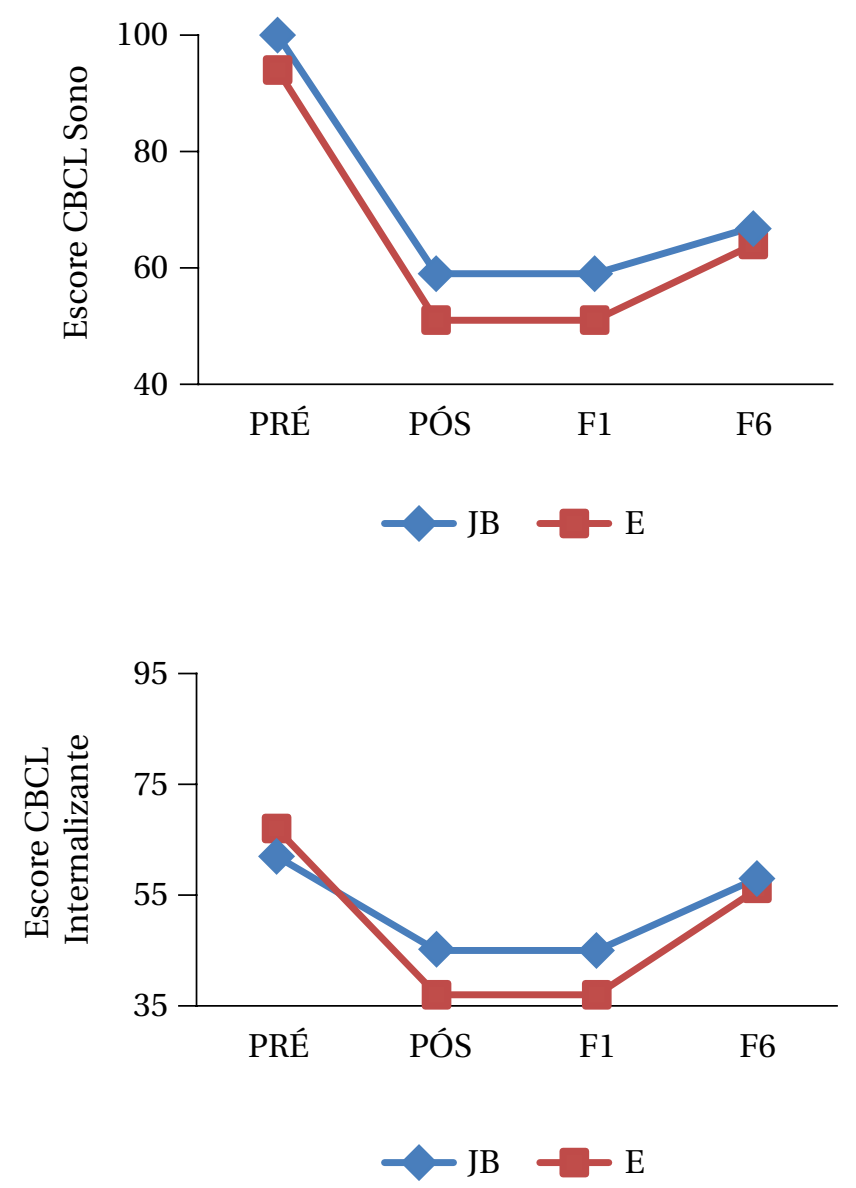

\section{Discussão}

Os resultados demonstram que a intervenção para insônia comportamental infantil por meio de orientação parental foi efetiva no caso relatado com irmãos gêmeos. Apesar da literatura internacional (Mindell et al., 2006) e pesquisa brasileira (Rafihi-Ferreira et al., 2019) relatarem a eficácia da intervenção comportamental em crianças pré-escolares, não são neles destacados a insônia comportamental infantil em gêmeos.

Após a intervenção, houve uma modificação na rotina pré-sono das crianças, a resistência em ir para a cama foi reduzida, elas deixaram de despertar a noite e procurar os pais e passaram a adormecer independentemente, sem a ajuda parental. A melhora foi mantida nos períodos de seguimento, com exceção da resistência em ir para a cama, que retornou para os gêmeos após seis meses do término do tratamento.
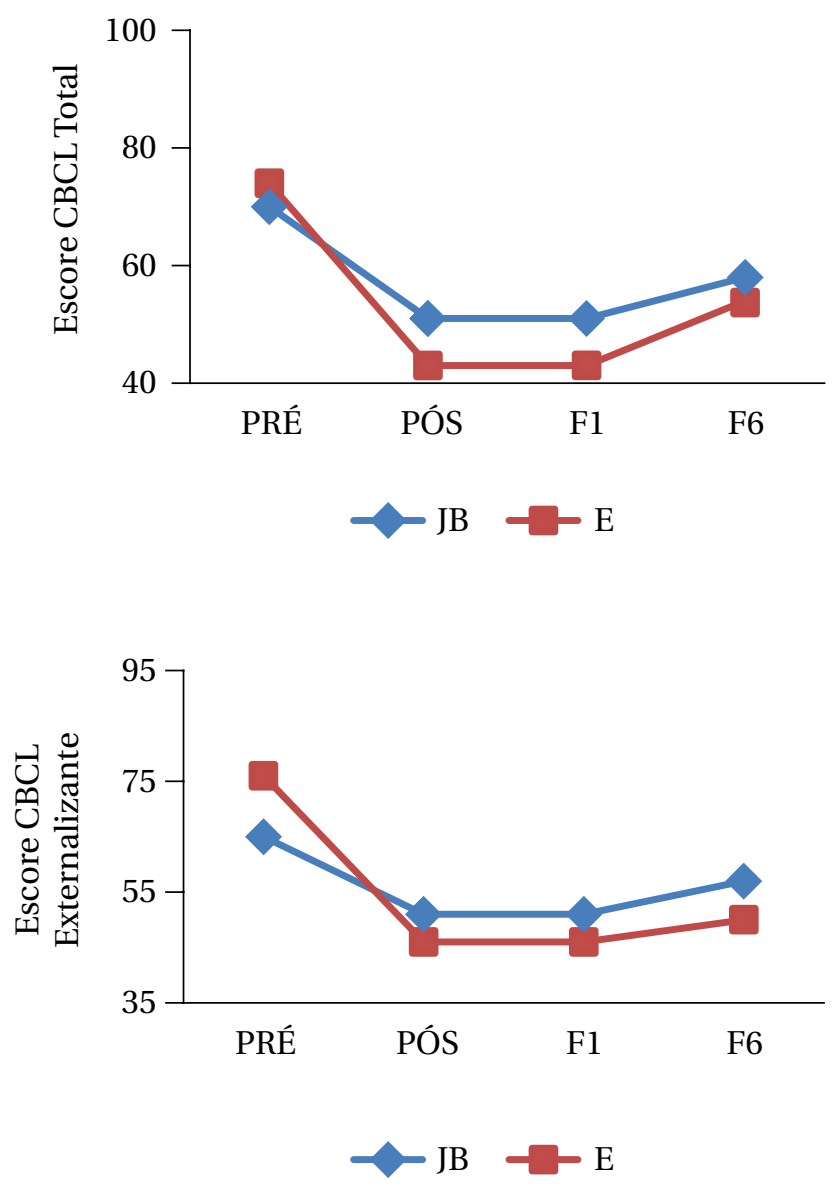

Figura 3

Escores do CBCL dos gêmeos nas Subescalas: Problemas de Sono, Total de Problemas de Comportamento, Problemas Internalizantes e Externalizante nas etapas pré, pós-intervenção e follow-up de um e seis meses. 
O fato de os cuidadores não manterem a rotina pré-sono dos filhos com consistência, isto é, nem sempre realizavam a rotina com as crianças com atividades calmas e voltaram a acrescentar o ato de assistir a TV momentos antes de dormir, são hipóteses para a resistência em ir para a cama ressurgir. Sobre este aspecto, Mindell, Telofski, Wiegand e Kurtz (2009) apontam que a consistência na rotina pré-sono pode reduzir a resistência em ir para a cama e melhorar a consolidação do sono das crianças.

Sobre a relação entre TV e sono na infância, o estudo de Mistry, Minkovitz, Strobino e Borzekowski (2007) indicou que o comportamento de assistir TV na primeira infância está associado com maiores problemas de sono, problemas de atenção e comportamento agressivo, avaliados pelo CBCL. A prática de assistir TV momentos antes de dormir também foi associado negativamente com quantidade de horas de sono em crianças (Thompson \& Christakis, 2005). De acordo com Thompson e Christakis (2005) a luz emitida pelos eletrônicos, antes de dormir, pode afetar o ciclo sono-vigília, por meio da supressão do hormônio de melatonina que é o hormônio que produz sonolência e que é bloqueado na presença de luz. Desta forma, supõe-se que assistir TV foi um dos fatores que contribuíram para os gêmeos voltarem a resistir em ir para a cama.

Outro fator que pode ter favorecido o retorno da resistência em ir para a cama no período de seguimento de seis meses, é que em algumas noites da semana os irmãos praticavam atividades vigorosas antes de adormecer. Segundo Allen, Howlett, Coulombe e Corkum (2016), as atividades vigorosas antes de dormir, podem interferir com a capacidade da criança para alcançar um estado relaxado e positivo, e, consequentemente interferir no processo de adormecer. Por isso destaca-se a importância da manutenção na rotina pré-sono e consistência parental, mesmo quando há melhora nos padrões de sono. De acordo com Meltzer e Mindell (2011) o foco na manutenção das rotinas pré-sono e a consistência do comportamental parental previne a ressurgência dos problemas de sono. A consistência do comportamento dos pais é fundamental para a rápida aprendizagem em crianças, pois quando o comportamento dos cuidadores é mantido, a criança consegue compreender a contingência que está em vigor. Além disso, segundo Patterson, Reid e Dishion (2002) a consistência das práticas educativas por parte dos pais constitui fator de proteção para o surgimento dos problemas de comportamento infantil. Em consonância com outros estudos (Brescianini et al., 2011; Fisher et al., 2012) este relato elucida a importância de considerar o ambiente familiar compartilhado como alvo de intervenção, ressaltando as rotinas e práticas de higiene do sono como ingredientes essenciais para a qualidade do sono e também para o comportamento diurno infantil.

Além do estabelecimento de rotinas, o procedimento de extinção possivelmente teve papel-chave na redução dos despertares e na independência dos gêmeos no momento de dormir. Ao reduzir o envolvimento parental momentos antes de dormir, as crianças tiveram a oportunidade de desenvolver habilidades de autorregulação que são importantes para o aprendizado em dormir independentemente. De acordo com Thomas et al. (2014), o suporte empírico e o sucesso nos resultados da técnica de extinção é justamente por esta alcançar o objetivo de promover o desenvolvimento de habilidades de autoacomodação na criança.

Sobre a prática de compartilhar a cama, Ferber (2006) aponta que ao dividir a cama, pais e filhos podem despertar mais e demorar mais para dormir e as crianças dormirem tarde em decorrência da rotina parental ou por ficarem mais agitadas em ambiente compartilhado. De fato, no presente estudo essas características foram encontradas, uma vez que os gêmeos despertavam mais, dormiam mais tarde e tinham menor quantidade de horas de sono quando compartilhavam a cama com os pais. Tais achados vão ao encontro do que a literatura aponta sobre a prática de dormir com os pais. Mindell, Sadeh, Kohyama e How (2010) reforçam que o compartilhamento da cama está associado com maior número de despertares e menor quantidade de horas de sono. Além disso, a criança pode associar o início do sono com a presença dos pais e só conseguir adormecer na presença destes, tendo dificuldade em dormir sozinha. Neste relato de caso, antes da intervenção, as duas crianças dormiam com os pais, na cama do casal, de modo que a presença do cuidador poderia estar associada com o início do sono. Depois das orientações, os gêmeos passaram a não mais compartilhar a cama com os pais, indicando que a intervenção foi efetiva para as crianças dormirem independentemente. 
Outro aspecto que merece destaque é que as crianças passaram a dormir lado a lado e não mais solicitar atenção parental. Por terem a mesma idade, os mesmos hábitos e dividirem o mesmo espaço, levanta-se a hipótese de que a companhia do irmão gêmeo pode ter contribuído para as crianças dormirem sem o auxílio parental. De acordo com Goldsmid e Féres-Carneiro (2011), as experiências compartilhadas entre irmãos exercem um papel importante para o desenvolvimento da relação fraterna, isto é, entre os irmãos, permitindo um maior distanciamento entre pais e filhos, o que fortalece capacidades e tolerâncias, incluindo conflitos, frustrações e capacidades para regulação, antecipação, planejamento e adaptação.

Além da melhora nos padrões de sono dos gêmeos, também foi observado melhora nos comportamentos diurnos, avaliados pelo CBCL. Houve redução nas pontuações dos problemas de comportamento externalizante, internalizante, total de problemas de comportamento e problemas de sono, que passaram de clínicos para normais depois da intervenção. A melhora de comportamentos diurnos após intervenção para insônia também já foi observada em outros estudos (Pinilla \& Birch, 1993; Rafihi-Ferreira et al., 2019), sem enfoque, contudo, na população de irmãos gêmeos. Mindell et al. (2006) indicam que um fator provável para estas melhoras em comportamentos diurnos é o aumento na quantidade e qualidade de sono que as crianças experimentam após um tratamento efetivo. Outro fator que merece destaque é que a intervenção envolve modificações dos comportamentos dos pais e do ambiente, e consequentemente interfere nas respostas das crianças. A consistência do comportamento parental no momento de dormir, por meio de estabelecimentos de limites e modificação do ambiente, por meio de rotinas, pode ser generalizada também para os comportamentos durante o dia. Uma hipótese é que os pais passaram a estabelecer rotinas e limites também no período diurno, o que pode ter contribuído para a melhora dos comportamentos. Vale lembrar, que a melhora do sono e do comportamento diurno dos gêmeos corrobora o que Thomas et al. (2014) salientam sobre os efeitos positivos da intervenção comportamental, que não produz efeitos negativos para as crianças, demostrando ser uma prática segura.

Embora efetivo, o presente relato apresenta limitações. Os dados aqui apresentados são baseados em relato parental. Além disso, não foram realizadas comparações com outros participantes. Devido a estes fatores, réplicas de casos como este são necessárias para controle de variáveis internas e externas, como também a combinação de relato parental com outras medidas de avaliação, como actigrafia ou registros de vídeo.

Os resultados apresentados neste estudo chamam atenção no efeito que a intervenção baseada na aprendizagem operante teve sobre o sono e comportamento das crianças. Este aspecto ressalta cada vez mais a importância de uma inserção maior da questão do sono na Psicologia, a partir de práticas preventivas e interventivas.

\section{Conclusão}

Pode-se concluir, que o manejo da insônia infantil por meio de orientação parental levou a melhora na qualidade de sono e nos comportamentos diurnos dos gêmeos. Por tratar-se de um relato de caso, esses resultados não podem ser generalizados para todas as crianças gêmeas que apresentam problemas comportamentais relacionadas ao sono. Para responder se de fato a intervenção comportamental para insônia infantil - que compreende orientações aos pais sobre o estabelecimento de rotina para dormir com reforço positivo e o uso da técnica de extinção - é efetiva em crianças gêmeas em idade pré-escolar e se esta intervenção pode beneficiar os comportamentos diurnos, são necessárias pesquisas com maior número de participantes, que compare crianças gêmeas e não gêmeas que foram e que não foram submetidas a intervenção ou investigações que utilizem o delineamento de sujeito único com linha de base múltipla.

O presente trabalho elucida o importante papel do comportamento parental na manutenção do comportamento infantil ao demonstrar que é possível modificar comportamentos inadequados em um curto espaço de tempo por meio de mudanças de respostas parentais, mesmo em casos que demandam maior cuidado e atenção, como com crianças gêmeas. Por fim, este relato ressalta a relevância da inserção do estudo do sono no curso de Psicologia, visto que os problemas de sono se configuram uma demanda frequente nessa faixa etária, que necessita de atenção profissional adequada para o seu manejo, inclusive em populações como a relatada neste caso. 


\section{Referências}

Achenbach, T. M., \& Rescorla, L. A. (2000). Manual for the Aseba preschool forms \& profiles. Burlington, VT: University of Vermont.

Allen, S. L., Howlett, M. D., Coulombe, J. A., \& Corkum, P. V. (2016). ABCs of sleeping: A review of the evidence behind pediatric sleep practice recommendations. Sleep Medicine Review, 29, 1-14. https://doi.org/10.1016/j. smrv.2015.08.006

Andrade, L., Martins, M. M., Angelo, M., \& Martinho, J. (2014). A família na vivência da gemilaridade: Revisão sistemática. Texto Contexto Enfermagem, 23(3), 758-766. https://doi.org/10.1590/0104-07072014002950013

Blunden, S. L., \& Chervin, R. D. (2010). Sleep, performance and behavior in australian indigenous and non-indigenous children: An exploratory comparison. Journal of Paediatrics and Child Health, 46(1/2), 10-16. https://doi.org/10.1111/j.1440-1754.2009.01610.x

Brescianini, S., Volzone, A., Fagnani, C., Patriarca, V., Grimaldi, V., Lanini, R. et al. (2011). Genetic and environmental factors shape infant sleep patterns: A study of 18-month-old twins. Pediatrics, 127(5), 1296-1302. https://doi.org/10.1542/peds.2010-0858

Byars, K. C., Yeomans-Maldonado, G., \& Noll, J. G. (2011). Parental functioning and pediatric sleep disturbance: An examination of factors associated with parenting stress in children clinically referred for evaluation of insomnia. Sleep Medicine, 12(9), 898-905. https://doi.org/10.1016/j.sleep.2011.05.002

David, D. L., Azevedo, E. C., Russi, E. M. S., Berthoud, C. M. E., \& Oliveira, A. L. (2000). Tríade de contato intimo: Apego entre mãe e filhos gêmeos. Revista de Biociências, 6(1), 57-63.

Didden, R., Sigafoos, J., \& Lancioni, G. E. (2011). Unmodified extinction for childhood sleep disturbance. In M. Perlis, M. Aloia, \& B. Kuhn (Orgs.), Behavioral treatments for sleep disorders: A comprehensive primer of behavioral sleep medicine interventions (pp. 257-263). London: Elsevier.

Ferber, R. (2006). Solve your child's sleep problems. New York, NY: Fireside.

Fisher, A., Jaarsveld, C. H. M., Llewellyn, C. H., \& Wardle, J. (2012). Genetic and environmental influences on infant sleep. Pediatrics, 129(6), 1091-1095. https://doi.org/10.1542/peds.2011-1571

Goldsmid, R., \& Féres-Carneiro, T. (2011). Relação fraternal: Constituição do sujeito e formação do laço social. Psicologia USP, 22(4), 771-187. https://doi.org/10.1590/S0103-65642011005000031

Gregory, A. M., Eley, T. C., O'Connor, T. G., Rijsdijk, F. V., \& Plomin, R. (2005). Family influences on the association between sleep problems and anxiety in a large sample of pre-school aged twins. Personality and Individual Differences, 39(8), 1337-1348. https://doi.org/10.1016/j.paid.2005.06.008

Kuhn, B. R. (2011). Behavioral protocol to promote independent sleep initiation skills and reduce bedtime problems in young children. In M. Perlis, M. Aloia, \& B. Kuhn (Orgs), Behavioral treatments for sleep disorders: A comprehensive primer of behavioral sleep medicine interventions (pp. 299-309). London: Elsevier.

Lytton, H., Singh, J. K., \& Gallagher, L. (1995). Parenting twins. In M. H. Bornstein (Org.), Handbook of parenting (pp. 185-208). Mahwah, NJ: Lawrence Erlbaum Associates.

Meltzer, L. J., \& Mindell, J. A. (2011). Graduated extinction: Behavioral treatment for bedtime problems and night wakings in young children. In M. Perlis, M. Aloia, \& B. Kuhn (Orgs), Behavioral treatments for sleep disorders: A comprehensive primer of behavioral sleep medicine interventions (pp. 265-273). London: Elsevier.

Meltzer, L. J. \& Mindell, J. A. (2014). Systematic review and meta-analysis of behavioral interventions for pediatric insomnia. Journal of Pediatric Psychology, 39(8), 932-948. https://doi.org/10.1093/jpepsy/jsu041

Mindell, J. A., Kuhn, B., Lewin, D. S, Meltzer, L. J., \& Sadeh, A. (2006). Behavioral treatment of bedtime problems and night wakings in infants and young children: An american academy of sleep medicine review. Sleep, 29(10), 1263-1276.

Mindell, J. A., Sadeh, A., Kohyama, J., \& How, T. H. (2010). Parental behaviors and sleep outcomes in infants and toddlers: A cross-cultural comparison. Sleep Medicine, 11(4), 393-399. https://doi.org/10.1016/j.sleep.2009.11.011.

Mindell, J. A., Telofski, L., Wiegand, B., \& Kurtz, E. S. (2009). A nightly bedtime routine: Impact on sleep in young children and maternal mood. Sleep, 32(5), 599-606. 
Mistry, K. B., Minkovitz, C. S., Strobino, D. M., \& Borzekowski, D. L. G. (2007). Children’s television exposure and behavioral and social outcomes at 5.5 years: Does timing of exposure matter? Pediatrics, 120(4), 762-769. https://doi.org/10.1542/peds.2006-3573

Oord, E. J. C. G., Koot, H. M., Boomsma, D. I., Verhulst, F. C., \& Orlebeke, J. F. (1995). A twin-singleton comparison of problem behaviour in 2-3-year-olds. Journal of Child Psychology and Psychiatry, 36(3), 449-458. https://doi.org/10.1111/j.1469-7610.1995.tb01302.x

Patterson, G., Reid, J., \& Dishion, T. (2002). Antisocial boys: Comportamento anti-social. Santo André, SP: ESETec.

Pires, M. L. N., Vilela, C. B., \& Camara, R. L. (2012). Desenvolvimento de uma medida de hábitos de sono e aspectos da prevalência de problemas comportamentais de sono na infância: Uma contribuição. In N. Silva Filho, D. P. S. A. Ribeiro, \& H. R. Rosa (Orgs.), Processos clínicos e saúde mental (pp. 167-192). São Paulo, SP: Cultura Acadêmica.

Pinilla, T., \& Birch, L. L. (1993). Help me make it through the night: Behavioral entrainment of breast-fed infants 'sleep patterns. Pediatrics, 91(2), 436-444.

Rafihi-Ferreira, R., Pires, M. L. N., \& Silvares, E. F. M. (2019). Behavioral intervention for sleep problems in childhood: A brazilian randomized controlled trial. Psicologia: Reflexão e Crítica, 32(1), 1-13. https://doi.org/10.1186/ s41155-019-0118-3

Rafihi-Ferreira, R., Silvares, E. F. M., Pires, M. L. N., Assumpção, F. B., Jr, \& Moura, C. B. (2016). Sono e comportamento em crianças atendidasem um serviço de psicologia. Psicologia: Teoria e Prática, 18(2), 159-172. https://doi.org/10.15348/1980-6906/psicologia.v18n2p159-172

Richman, N., \& Graham, P. (1971). A behaviour-screening questionnaire for use with three-year-old children. Journal of Child Psychology and Psychiatry, 12(1), 5-33. https://doi.org/10.1111/j.1469-7610.1971.tb01047.x

Thomas, J. H., Moore, M., \& Mindell, J. A. (2014). Controversies in behavioral treatment of sleep problems in young children. Sleep Medicine Clinic, 9(1), 251-259. https://doi.org/10.1016/j.jsmc.2014.02.004

Thompson, D. A., \& Christakis, D. A. (2005). The association between television viewing and irregular sleep schedules among children less than 3 years of age. Pediatrics, 116(4), 851-856. https://doi.org/10.1542/peds.2004-2788

Tikotzky, L., \& Sadeh, A. (2010). The role of cognitive-behavioral therapy in behavioral childhood insomnia. Sleep Medicine, 11(7), 686-691. https://doi.org/10.1016/j.sleep.2009.11.017

\section{Renatha El Rafihi-Ferreira}

Psicóloga. Mestre em Análise do Comportamento pela Universidade Estadual de Londrina (UEL), Londrina - PR. Brasil. Doutora e Pós-doutoranda em Psicologia Clínica pela Universidade de São Paulo (USP), São Paulo - SP. Brasil.

E-mail: renatha.rafihi@hc.fm.usp.br

https://orcid.org/0000-0002-2212-4608

\section{Maria Laura Nogueira Pires}

Psicóloga. Mestre em Psicobiologia. Doutora em Ciências pela Universidade Federal de São Paulo (Unifesp), São Paulo - SP. Brasil. Pós-Doutora pelo Departamento dos Transtornos do Sono e do Humor da Oregon Health Sciences University, EUA. Docente da Universidade Estadual Paulista Júlio de Mesquita Filho (Unesp), Assis - SP. Brasil.

E-mail:laurapires@assis.unesp.br

https://orcid.org/0000-0001-8050-1187

\section{Edwiges Ferreira de Mattos Silvares}

Psicóloga. Mestre e Doutora em Psicologia Experimental pela Northeastern University, EUA/Universidade de São Paulo (USP), São Paulo - SP. Brasil. Livre-docente, Professora titular e colaboradora sênior da USP.

E-mail: efdmsilv@usp.br

https://orcid.org/0000-0002-4958-7549 
Endereço para envio de correspondência:

Renatha El Rafihi-Ferreira. Avenida Professor Mello Morais 1721. Butantã. CEP: 05508-900.

São Paulo - SP. Brasil.

Recebido 07/03/2017

Reformulado 22/04/2019

Aceito 29/04/2019

Received 03/07/2017

Reformulated 04/22/2019

Approved 04/29/2019

Recibido 07/03/2017

Reformulado 22/04/2019

Aceptado 29/04/2019

Como citar: Rafihi-Ferreira, R.; Pires, M. L. N. e Silvares, E. F. M. (2019). Orientação Parental para Insônia Infantil em Irmãos Gêmeos: Um Relato de Caso. Psicologia: Ciência e Profissão, 39, 1-14. https://doi.org/10.1590/1982-3703003176715

How to cite: Rafihi-Ferreira, R.; Pires, M. L. N. e Silvares, E. F. M. (2019). Parental Guidance for Childhood Insomnia in Twins: A Case Report. Psicologia: Ciência e Profissão, 39, 1-14. https://doi.org/10.1590/1982-3703003176715

Cómo citar:Rafihi-Ferreira, R.; Pires, M. L. N. e Silvares, E. F. M. (2019). Orientación Parental para El Insomnio Infantil en Hermanos Gemelos: Un Informe de Caso. Psicologia: Ciência e Profissão, 39, 1-14. https://doi.org/10.1590/1982-3703003176715 